LA-UR- $1 /-0 / 630$

Approved for public release: distribution is unlimited.
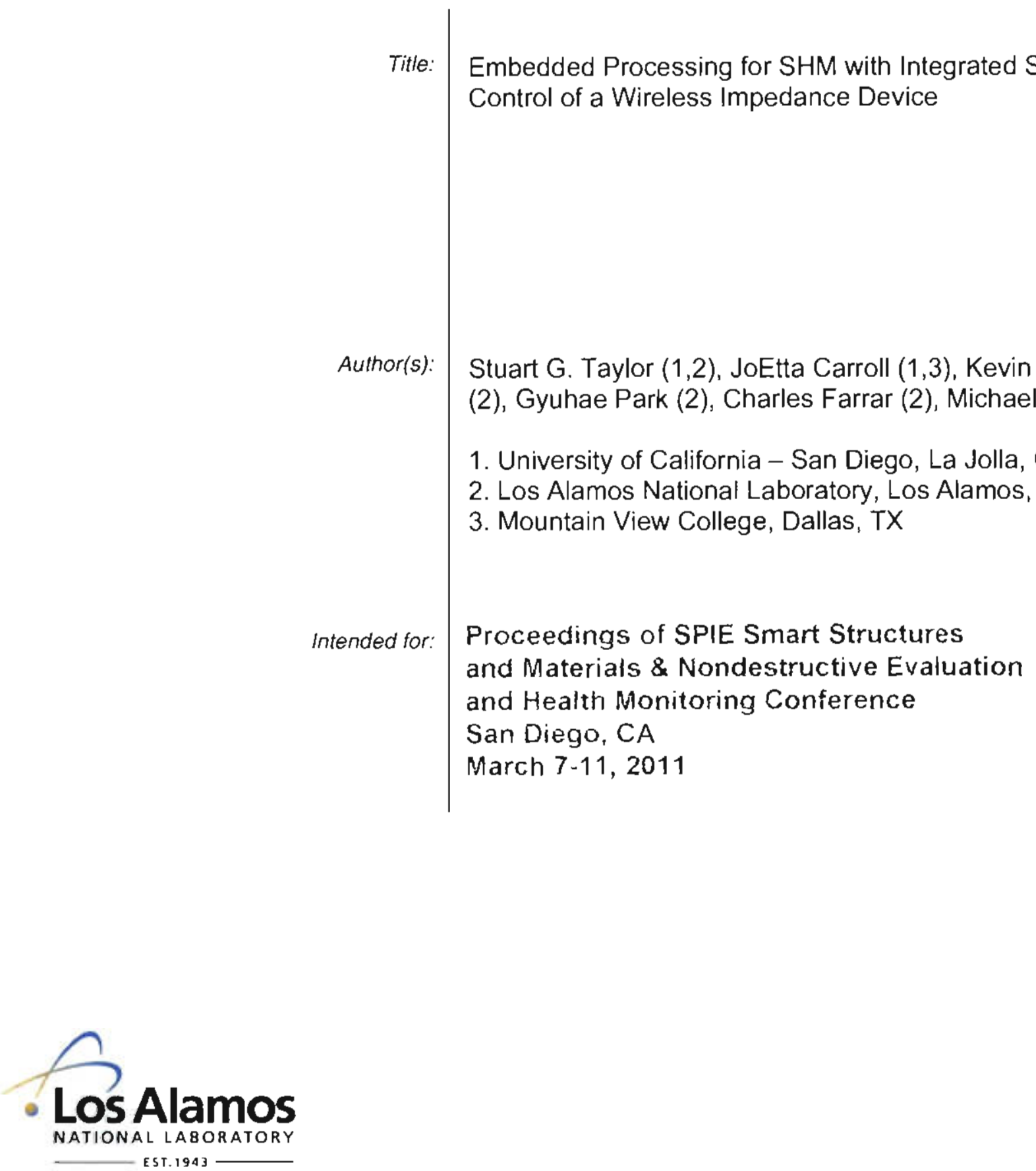

Los Alamos National Laboratory, an affirmative action/equal oppontunity employer, is operated by the Los Alamos National Security, LLC for the National Nuclear Security Administration of the U.S. Department of Energy under contract DE.AC52-06NA25396. By acceplance of this article, the publisher recognizes that the U.S. Government relains a nonexclusive, royalty-free license to publish or reproduce the published form of this contribution, or to allow olhers to do so, for U.S. Government purposes. Los Alamos Nalional Laboratory requests that the publisher identify this article as work performed under the auspices of the U.S. Department of Energy. Los Alamos National Laboratory strongly supports academic freedom and a researcher's right 10 publish; as an institution, however, the Laboratory does not endorse the viewpoint of a pubfication or guarantes its technical correciness. 


\title{
Embedded Processing for SHM with Integrated Software Control of a Wireless Impedance Device
}

\author{
S. G. Taylor ${ }^{1,2}$, J. Carroll ${ }^{3}$, K. M. Farinholt ${ }^{4}$, G. Park ${ }^{1, *}$, C. R. Farrar' ${ }^{1}$, M. Todd ${ }^{2}$ \\ 1. The Engineering Institute, Los Alamos National Laboratory, Los Alamos, NM \\ 2. University of California - San Diego, La Jolla, CA \\ 3. Mountain View College, Dallas, TX \\ 4. Applied Engineering Technology, Los Alamos National Laboratory, Los Alamos, NM
}

\begin{abstract}
Wireless sensor nodes with impedance measurement capabilities, often based on the Analog Devices AD5933 impedance chip and Atmel's 8-bit ATMega 1281 microcontroller, have been demonstrated to be effective in collecting data for localized damage detection (such as for loose bolt detection) and for sensor self-diagnostics. Previouslydeveloped nodes rely on radio telemetry and off-board processing (usually via a PC) to ascertain damage presence or sensor condition. Recent firmware improvements for the wireless impedance device (WID) now allow seamless integration of the WID witb SHMTools and MFUSE, an open-source function sequencer and SHM process platform for Matlab. Furthermore, SHM processes developed using mFUSE can be implemented in hardware on the WID, allowing greater autonomy among the sensor nodes to identify and report damage in real time. This paper presents the capabilities of the newly integrated hardware and software, as well as experimental validation.
\end{abstract}

Keywords: Embedded Sensing, Wireless Sensor Node, Sensor Diagnostics, Structural Health Monitoring

\section{INTRODUCTION}

Structural health monitoring (SHM) is the process of detecting damage in structures. The goal of SHM is to improve the safety and reliability of aerospace, civil, and mechanical infrastructure by detecting damage before it reaches a critical state. A more detailed general discussion of SHM can be found in Farrar et al. (1999). The implementation of SHM is an integrated paradigm of networked sensing and actuation, data internogation, and statistical assessment that treats structural health assessments in a systematic way. An appropriate sensor network is always required as a first line of attack in observing the structural system behavior in such a way that suitable signal processing and damage-sensilive feature extraction from the measured data can be performed efficiently.

In order for any SHM system to be successful, there must be a reliable stream of operational data available for analysis, and little or no intervention should be required on the part of engineers to obtain such data. This study addresses the reliability and longevity issues surrounding the collection of data from a remote civil infrastructure site when there are long intervals between on-site maintenance of the sensing and data acquisition systems. Such a system requires a robust sensor network.

Sensor networks contain four main components: the sensing mechanism, computation, telemetry, and power management (Farrar ot al. 2006). In many SHM applications, including the one presented in this work, an actuation component is also required. The most common general architecture that integrates these components is a conventional wired sensor network. Here, individual sensing components typically stand alone and are wired to a centralized data processiag and multiplexing unit. Each sensor is independent of other sensors in the network, and controlled synchronized interrogation of the entire network is achieved only through the central unit. Limitations of such systems are that they are difficult to deploy in a retrofit mode because they usually require a power source, which is not always

* gpark@alan].com 
readily available in existing structures. Also, these systems are one-point failure sensitive, with a single one wire being as long as a few hundred meters. The deployment of such system can be challenging with much of the installation time taken by wires and cables (Lynch et al. 2003).

While the majority of permanently installed sensor networks today employ this wired architecture, development and deployment of wireless sensor networks has exploded in recent years. Most wireless sensor network paradigms fall into one of the categories outlined by Farrar et al. (2006). However, while much exploratory work has been done to assess the feasibility of wireless networks for SHM, relatively little has been done toward a permanently installed wireless sensor network for SHM.

This paper presents improvements on existing implementation of wireless sensor nodes by providing integrated software control using the SHMTools package. The SHMTools package can be used to control the sensor hardware directly by requesting measurements and performing subsequent analysis and classification of the received data, or by developing a process which can be optimized for the sensor node and loaded onto it for autonomous operation.

\section{WIRELESS SENSOR NODE HARDWARE}

The wireless impedance device (WID) was originally developed based on capabilities demonstrated in previous studies of the impedance-based structural health monitoring method [1]. The impedance method uses high-frequency vibrations to momitor for changes in structural impedance that would indicate damage. The impedance method can be implemented with relatively low power compared to other active-sensing SHM techniques such as Lamb wave-based methods. The impedance method also has applications in sensor self-diagnostics to determine the operational status of piezoelectric active-sensors used in SHM [2].

Three generations of the WID have been developed and field-tested by our research team [3-5]. The WID2 was developed to address some of the limitations of the previous version, which could monitor only a single sensor, had limited triggering capabilities, and used telemetry components with high power demands. The WID3 has further extended capabilities with advanced commumications, increased triggering options, local data storage, and multiple powering options including a variety of energy harvesting sources. The WID3 can self-configure into a network with neighboring sensor nodes at fixed time intervals or in the presence of a 'mobile-agent' that interrogates the sensor network.

In addition to improving the capabilities of the previous WID versions, the WID3 has been designed to function as part of a modular hardware platform that incorporates other sensing modalities on separate boards, including time-domain measurements. By combining modules, resources such as telemetry, processing, data storage, and respective measurement capabilities of each module can be shared, resulting in a highly functional sensor node. This integrated sensor node combines both actuation and sensing capabilities in a single package with the ability to implement multiple SHM techniques for the rapid health assessment of civil, aerospace and mechanical infrastructure.

The major hardware components of the WID3 are shown in Fig. 1. The WID3 uses a ZigBit module which integrates an Atmel ATmega1281v microcontroller ( $\mu \mathrm{Cu})$ with an Atmel AT86RF230 radio in a single integrated circuit (IC). The $\mu \mathrm{Cu}$ is part of Atmel's 8-bit AVR line, and it contains $128 \mathrm{kB}$ of flash memory for algorithm and code storage. It also contains $8 \mathrm{kB}$ of SRAM for program execution. The AT86RF230 is an 802.15.4 compliant radio, and it uses an open media access control (MAC) table distributed by ZigBit. The availability of the MAC table facilitates programming for robust data transmission. The AT86RF230 has very low energy requirements and requires few external components, making it particularly attractive for an SHM device. 

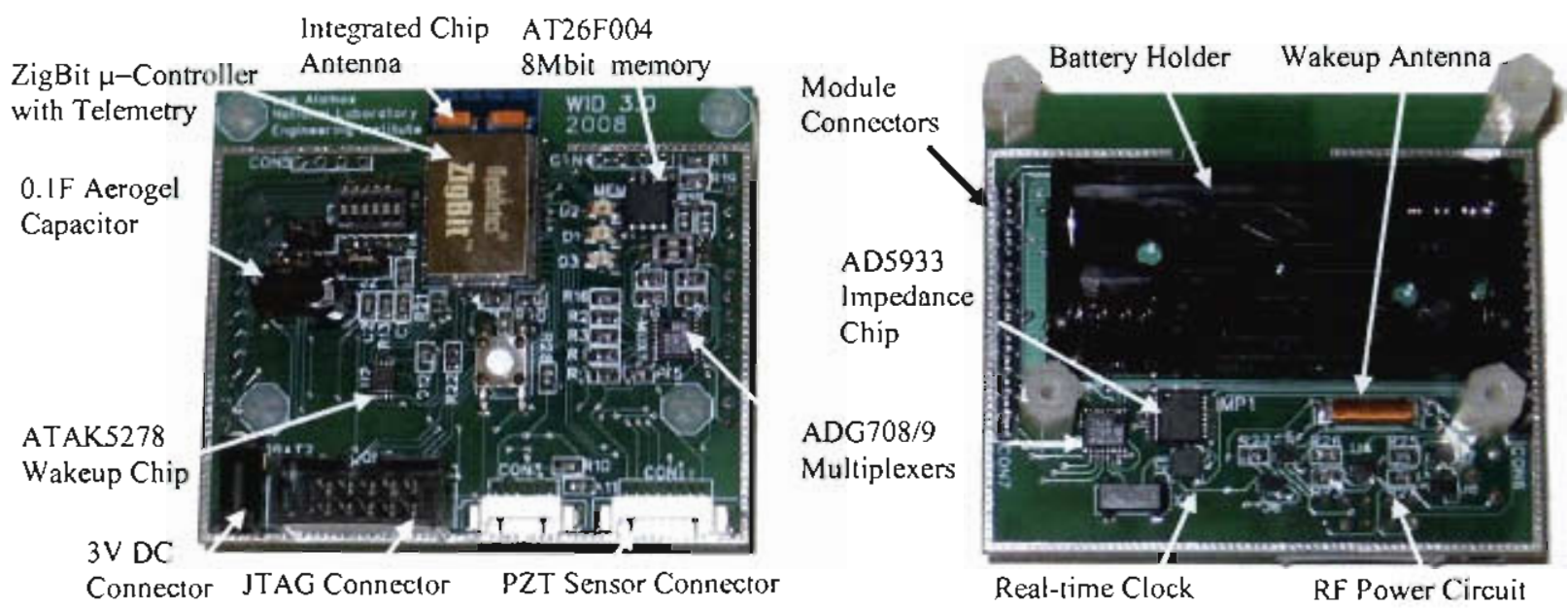

Fig. I. Major components of the WID3

The key measurement component the WID3 is the AD5933, an IC for impedance measurement. This IC has the ability to measure electrical impedance up to $100 \mathrm{kHz}$. The AD5933 can only measure a single channel, but the WID3 is equipped with two low-power and low-resistance multiplexers, which are indicaled in Fig. l. Each multiplexer has eight total inputs, providing for four impedance measurement ranges and the ability to measure seven sensors. One of the sensor ports is dedicated to a calibration cycle, reducing the number of sensor channels from eight to seven.

There are two main options for data storage on the WID3: internal EEPROM on the ATmega128 Iv, and a flash memory module, the Atmel AT26F004. The data storage available in these locations is $8 \mathrm{kB}$ and $512 \mathrm{kB}$, respectively. The WID3 has very low maximum power consumption in spite of the active nature of its measurements. Operating at $3 \mathrm{~V}$, the WID3 takes 16 seconds to measure four sensors with 100 points and four averages per point. With data reduction, only a few seconds would be required to transmit the data, or a few microseconds to store the data locally. The current draw can also be reduced to approximately $0.01 \mathrm{~mA}$ with proper use of sleep modes. With theșe steps, the WID3 could take, analyze, record and send one measurement per day for over 5 years on two conventional AA lithium batteries. At this extremely low power level, the WID3 could also be powered by a wide range of energy harvesting methods.

The WD3 can be woken from sleep states in two ways. The WID3 includes a low frequency wake-up chip, the Atmel ATA5283, which momitors for a $125 \mathrm{kHz}$ wake-up signal. This momitoring occurs at very low current draw $(0.1 \mu \mathrm{A})$ and shor range ( $2.5 \mathrm{~m})$. The chip and inductor coil antenna are indicated in Fig. 1. This wake-up method would be used by a mobile-agent for recording on-demand measurements. Secondly, an internal timer in the ATmegal281 v can wake up the WID3 at intervals on the order of a few seconds to a few weeks. With these solutions available, the WDD could rut in low duty cycle operation for decades on a limited power supply.

\section{SHMTOOLS SOFTWARE}

The software package - SHMTools - is the beginoing of a larger effort to collect and archive proven approaches to SHM for re-use by the research community. It is designed to facilitate the rapid assembly of custom SHM processes, provides a set of standard datasets5 on which to quickly evaluate the assembled processes, and provides a set of embeddable functions that are made easy to port to one's desired hardware platform. SHMTools is the result of a large collaborative effort between the Engineering Institute of Los Alamos National Laboratory (LANL), the Structural Engineering and the Computer Science departments at the University of California, San Diego.

The algorithms provided by SHMTools are organized into a set of standardized modules of MATLAB code covering three of the main stages of statistical pattern recognition as applied to SHM: data acquisition, feature extraction, and feature analysis for damage identification. Input and output parameters are standardized so that custom SHM processes are easily assembled by merely specifying a set of functions from each of these modules. Assembly routines are provided to further simplify the task. The mam assembly routine is a JAVA GUI (mFUSE) which allows functions to be dragged and dropped into a sequence to form a complete SHM algorithm. Variable types, values, and descriptions are 
displayed and functions are easily connected together by dragging output variables from one to the input variables of another, thus allowing for seamless data transfer. Once the algorithm is assembled, it may be run in its entirety, or selected functions can be run as needed. Algorithms can then be saved and restored for future manipulation or data interrogation. This assembly process is described in detail in Section 2 along with simple examples.

A subset of the functions in SHMTools is embeddable, can be cross-compiled from Matlab into generic "C" programs using the Matlab Real-time Workshop. We emphasize that this subset is independent of the target hardware and in fact can be compiled to virtually any platform using the appropriate cross-compiler. Various issues have to be taken into consideration. Most importantly, we have to ensure that the cross-compiled code is adequately optimized for the targeted bardware, and we have to ensure that there are enough hardware resources such as memory to properly run the embedded code on the hardware.

\section{CIVIL INFRASTRUCTURE TESTING RESULTS}

This section presents test results from the Alamosa Canyon Bridge, located in southern New Mexico, USA. This bridge has been decommissioned and made available by the New Mexico Department of Transportation as a test-bed for structural health inonitoring systems. The bridge has traditional steel girder construction with a reinforced concrete deck approximately $25 \mathrm{~cm}$ thick.

In this test, a total of 14 sensors were installed on two adjacent joints. One instrumented joint is on the west side (exterior) of the bridge, and the other joint is on the interior of the bridge. Each joint is bolted together with 14 bolts. A photograph of an instrumented bolted joint with an installed WID3 is shown in FIGURE. A diagram of the bolt pattern and sensor installation detail is shown in Fig. 2. The bolts are numbered vertically beginning with bolt 1 on the upper left of the front of the joint. The solid fill indicates a PZT installed on the nut (front side) or bolt head (rear side), and a circle indicates a PZT installed on the vertical surface adjacent to the bolt.

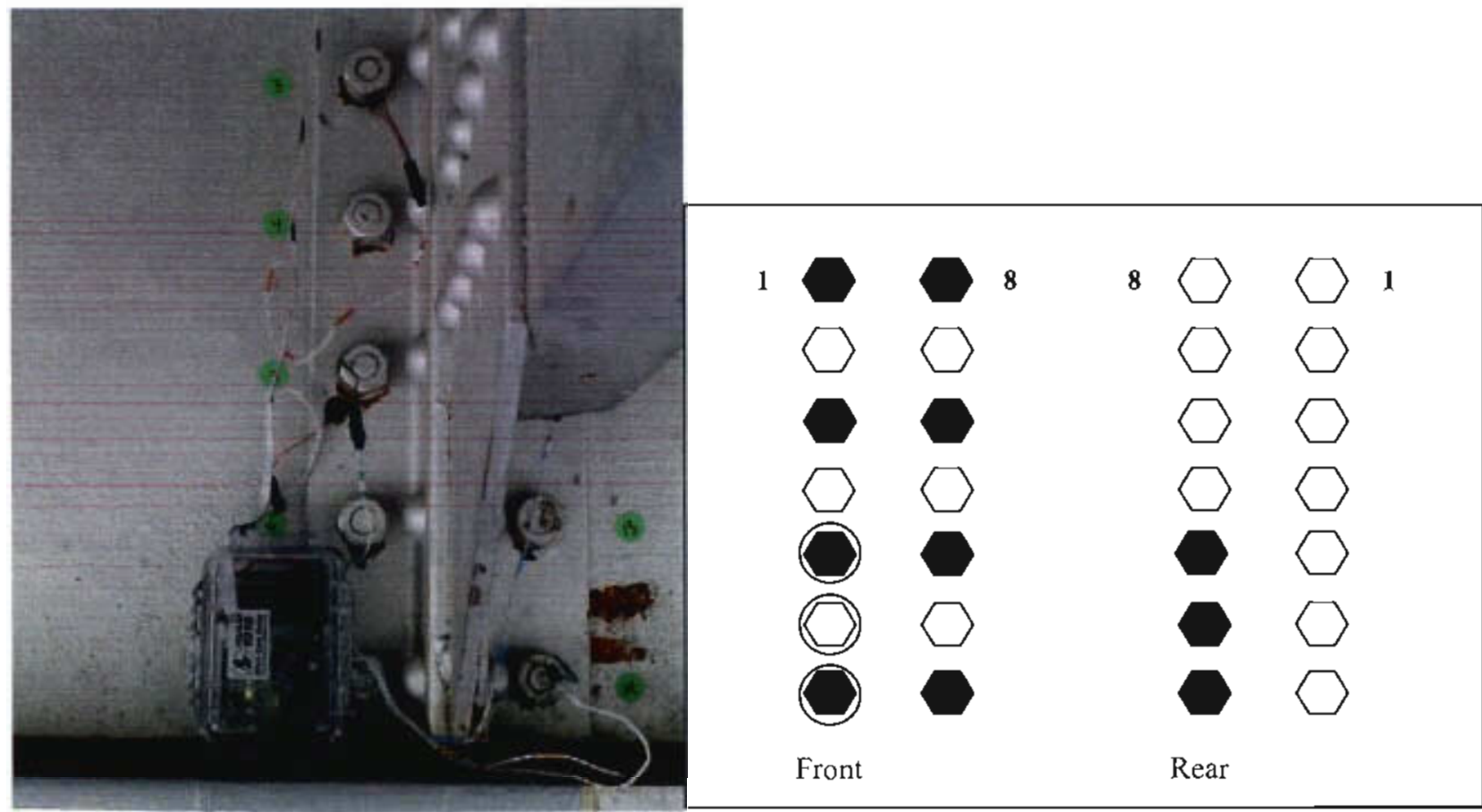

Fig. 2. Instrumented bolts with WID3 (1eft) and bolt pattern and instrumentation diagram (right)

Damage was introduced to the bridge in the form of loosening the instrumented nuts. Detection of bolted joint preload loss has been well demonstrated (Mascarenas et al. 2009, Overly et al. 2008, Taylor et al. 2009); it is not the goal of this study to demonstrate the ability to detect loss of bolt preload, but to investigate the viability of a permanently installed SHM system in a remote location. However, it is useful to demonstrate the ability of the SHM system to detect a given type of damage reliably prior to testing the longevity of a permanently installed SHM system. 
Selecled damage identification results are shown in Fig. 3; the left plot shows results from bolt 3 on the inner joint, while the right plot shows results from bolt 14 on the inner joint. In each case, impedance measurements were taken using the WID3 over a range of 80 to $100 \mathrm{kHz}$. Initial baseline measurements were made followed by damaged state measurements in two states: State 1, wherein the nut was just broken loose, but could not spin freely; and State 2, wherein the nut was completely loose and could spin freely. The nut was then re-tightened and another baseline measurement was taken.

In the case of bolt 3, shown in Fig. 3 (lefi), the second baseline measurement retumed to the same general wavefom as the first, and the damaged State waveforms are identifiable as damaged by peaks at around $88.5 \mathrm{kHz}$ and $86.5 \mathrm{kHz}$ for State 1 and State 2, respectively. In the case of bolt 14, shown in Fig. 3 (right), the second baseline measurement again returned to the same general waveform as the first, and the damaged State waveforms are identifiable as damaged by peaks at around $86.5 \mathrm{kHz}$ and $88 \mathrm{kHz}$, respectively.
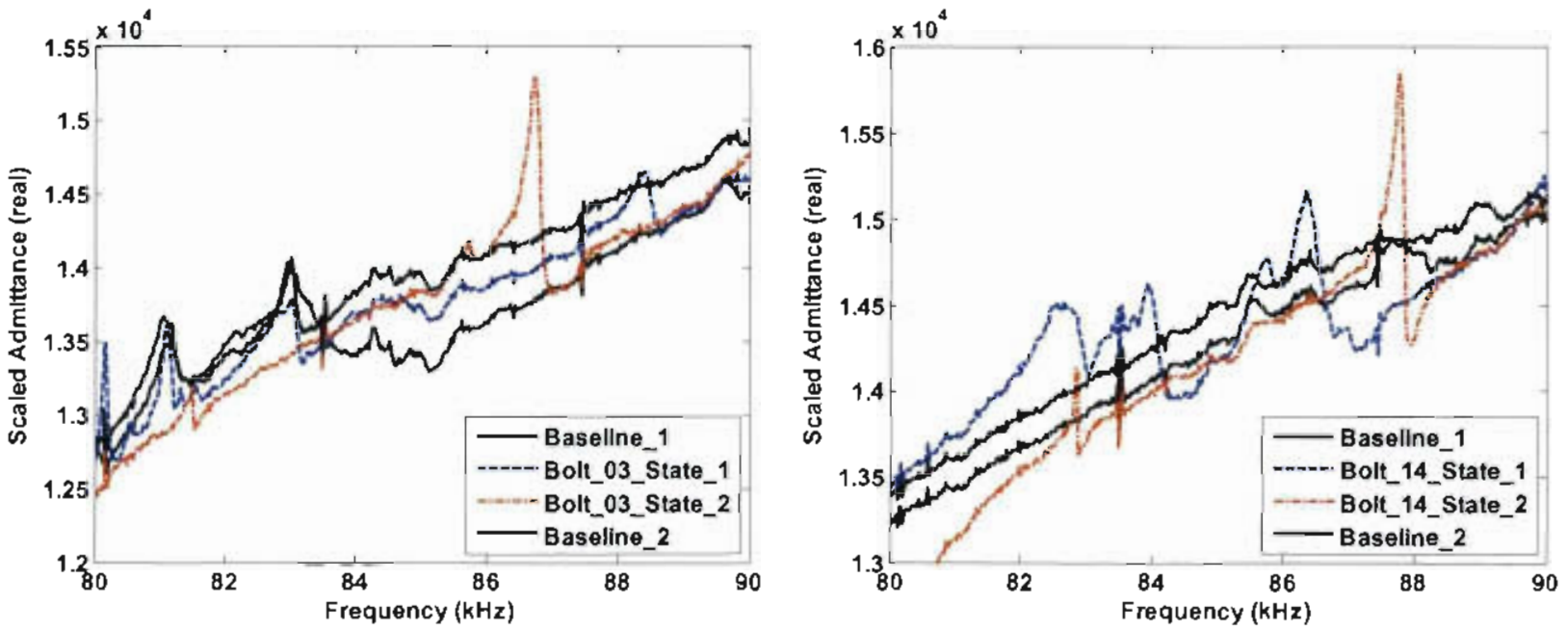

Fig. 3. Experimental data collected by the WID3 for inner joint bolt 3 (left) and 14 (right) in sevcral states

\section{SENSOR DIAGNOSTICS}

Any permanently installed SHM system will require the ability to accurately monitor the bealth of the sensors themselves, so that deviations from baseline measurements can be confidently attributed to structural changes rather than sensor malfunction. This section presents the sensor diagnostic capability of WID3, which is able to perform sensor diagnostics to determine whether the installed sensors/acruators are operational.

Such validation is critical during the SHM system operation, and arrays of lead zirconate titanate (PZT) active-sensors lend themselves particularly well to diagnostic interrogation regardless of the condition of the structure on which they are installed. The basis of this method is to traek the capacitive value of PZT transducers, which manifests itself in the imaginary part of the measured electrical admittance (Park et al. 2006a, 2006b). Both degradation of the mechanical/electrical properties of a PZT transducer and the bonding defects between a PZT patch and a host structure can be identified using this process. However, it should also be noted that temperature variations can manifest themselves in similar ways as sensor failures. In order to maintain robustaess to lemperature fluctuations, the authors have developed an efficient signal processing tool that enables the identification of a sensor validation feature that can be obtained instantaneously without relying on pre-stored baselines while remaining immune to temperature variations (Overly et al. 2009). The theoretical base of the sensor diagnostics and the signal processing tools is detailed in Park et al. (2009) and Overly et al. (2009). These diagnostics tools are incorporated in the SHMTools software package, currently under development by the authors. These tools were extended to utilize data collected with the WID3 system.

A sensor diagnostics plate, shown with the WID3 in Fig. 4, was constructed to demonstrate the sensor diagnostics capability. Twelve circular piezoelectric patches were mounted using super-glue on one surface of an Aluminum plate $(30 \times 30 \times 1.25 \mathrm{~cm}$ ). Each circular PZT measures $5.5 \mathrm{~mm}$ in diameter and $0.2 \mathrm{~mm}$ in thickness. Patches were given different bonding conditions, including perfect bonding, de-bonding, and sensor breakage. Six patches were bonded perfectly, three of them partially bonded with $25 \%, 50 \%$, and $75 \%$ de-honded area, and the remaining three were 
fractured with $25 \%, 50 \%$, and $75 \%$ sensor loss. After installation, admittance measurements were taken using the WID3 in the frequency range of $5-30 \mathrm{kHz}$ for each PZT patch.

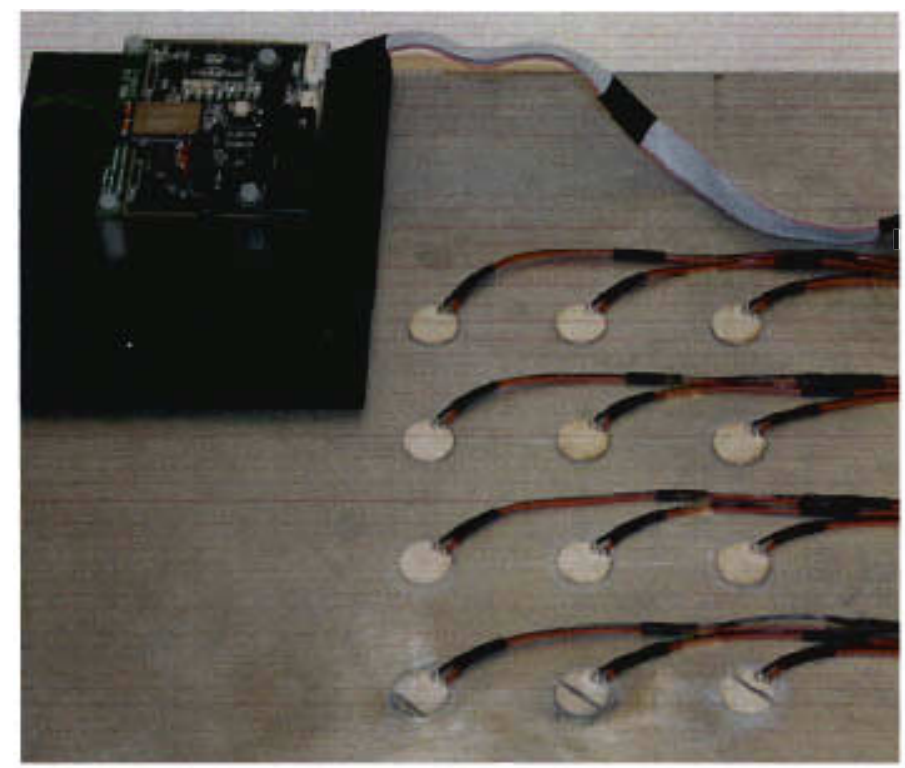

Fig. 4. Sensor Diagnostics demonstration plate with healthy, de-bonded, and broken sensors

The experimental sensor diagnostic results are illustrated in Fig. 5 and Fig. 6. For both measurement sets, the WID3 was connected to five healthy sensors and two faulty sensors. With de-bonded sensors, the slope of the measured admittance is greater than that for perfectly bonded sensors. As the de-bonded area increases, there is a corresponding increase in the slope, which is visible in Fig. 5 (left). With broken sensors, the slope of the measured admittance is less than that for perfectly bonded sensor, a result of the reduced capacitance of the now smaller sensor. In Fig. 5 (right), one can observe that the downward shift of the slope of the imaginary admittance is proportional to the breakage percentage. In Fig. 6 the results from the SHMTools sensor diagnostics functions are illustrated using data collected by the WID3. All the sensor conditions were correctly identified using the baseline-free algorithm developed by Overly et al. [6].
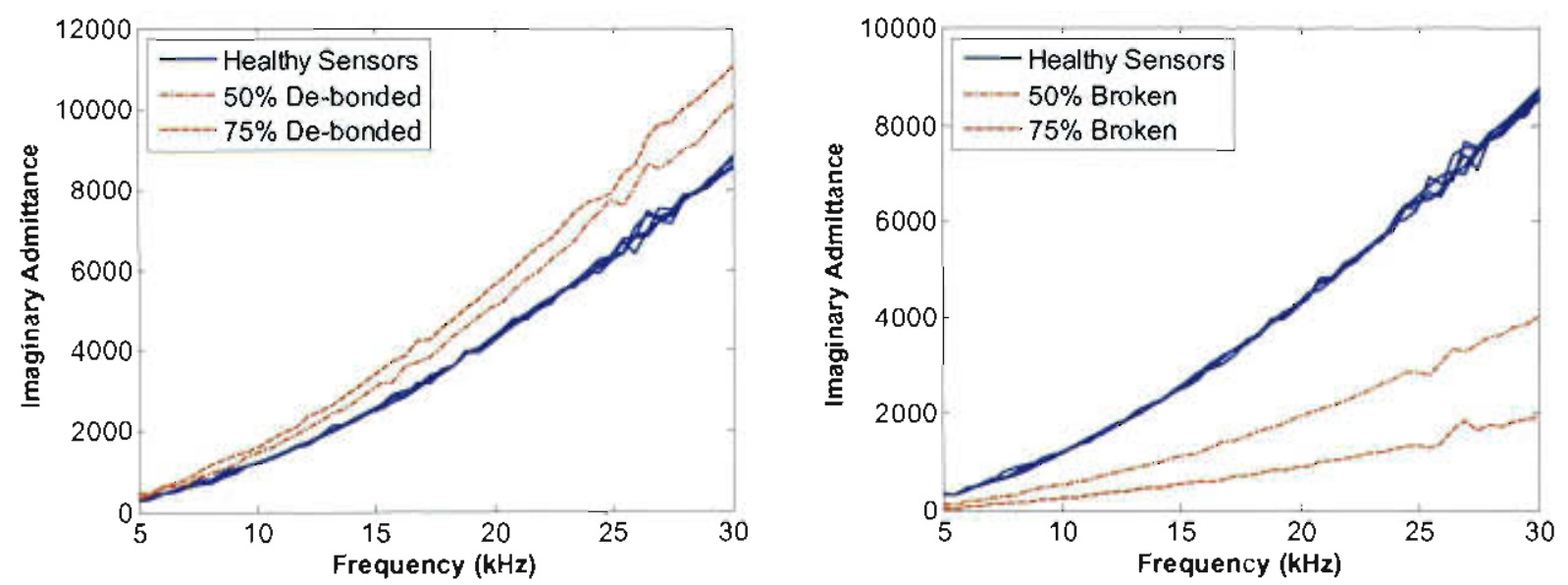

Fig. 5. Raw admittance data collected with the WID3 for de-bonded sensors (left) and broken sensors (right) 

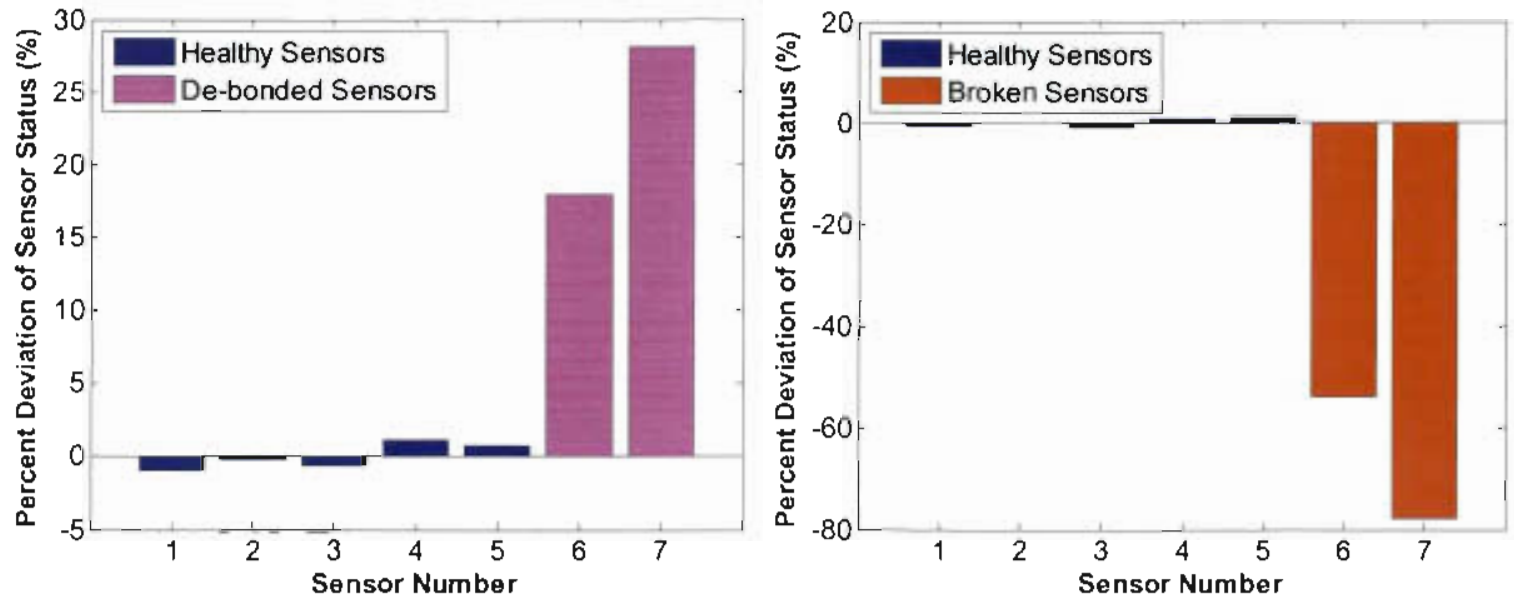

Fig. 6. Auto-classification results from SHMTools for de-bonded sensors (left) and broken sensors (right)

\section{ALGORITHM EMBEDMENT}

The SHMTools software package is designed to aid the transition from PC prototyping to embedded software. Key functions from the SHMTools library are wrilten using the Matlab Embedded Subset. For this subsel of functions and operators, Matlab provides equivalent efficied C-code. This allows Matlab functions making use of this subset to be automatically translated into $\mathrm{C}$ and in turn cross-compiled into stand-alone libraries for embedded systems.

The researcher first prototypes a potential SHM sequence on the PC through traditional coding or using the mFUSE: graphical interface. By prototyping within Matlab, the user has the freedom to explore different sequence possibilities, modify code, and tweak function parameters in order to maximize performance, all through Matlab's flexible, datacentric interface.

Once a potential sequence has been constructed and an initial set of parameters have been established, there are a number of ways to port the code to the embedded system with varying levels of sophistication. The most simple is to cross-compile the translated code to the embedded system. This step would typically require an input-outpur wrapper sitting around the Feature Extraction and Pattern Classification steps an SHM process. This wrapper would pass acquired data as an input to the cross-compiled SHMTools sequence and pass the resulting output to a PC or base station, allowing assembled sequences to be swapped in and out of the hardware with a fixed set of code.

\section{SUMMARY}

This paper has reviewed the capabilities of the Wireless Impedance Device (WID) and presented some new functionality utilizing software control of the WID using SHMTools and mFUSE. A path forward to implement sequences developed in mFUSE directly on the WID was described, which would enable mFUSE to be used as a firmware development tool for embedded SHM and on-line monitoring of civil infrastructure. Certain applications, including monitoring of bolted joints in bridge structures, were also discussed.

\section{REFERENCES}

[1] G. Park, et al., "Overview of Piezoelectric Impedance-Based Health Monitoring and Path Forward," Shock \& Vibration Digest, vol. 35, pp. 451-463, 2003.

[2] G. Park, et al., "Piezoelectric Active Sensor Self-Diagnostics Using Electrical Adnittance Measurements," Journal of Vibration and Acoustics, vol. 128, pp. 469-476, 2006.

[3] D. L. Mascarenas, et al., "Development of an impedance-based wireless sensor node for structural healtb monitoring," Smart Materials and Structures, p. 2137, 2007. 
[4] T. G. S. Overly, et al., "Development of an extremely compact impedance-based wireless sensing device," Smart Materials and Siructures, p. $065011,2008$.

[5] S. Taylor, el al., "Multi-scale wireless sensor node for health monitoring of civil infrastructure and mechanical systems," Smart Structures and Sysiems, vol. 6, pp. 661-673, 2010.

[6] T. G. Overly, er al. "Piezoelectric Active-Sensor Diagnostics and Validation Using Instantaneous Baseline Data," Sensors Journal, IEEE, vol. 9, pp. 1414-1421, 2009. 